\title{
New Approach about the Signaling Crosstalk between IQGAPs/ NF- $\kappa$ B/IL-8 and PDCD5/p53/TRAIL Pathways that Modulate Malignant Transformation in Hepatocellular Carcinoma
}

\author{
Khairy M A Zoheir*, Mohamed A Abdelhafez, Ahmed M Darwish, Karima F \\ Mahrous
}

\begin{abstract}
Objective: We aimed to investigate the signalling crosstalk of TNF-related apoptosis-inducing ligand, TRAIL death receptors, tumour protein p53, and programmed cell death (PDCD5) with IQGAPs. Also, we targeted the crosstalk between IQGAPs genes with decoy receptors, nuclear factor kappa-light-chain-enhancer of activated B cells (NF-kB), and interleukins -8 (IL-8) and its receptor genes in a designed model of hepatocellular carcinoma induced in male Balb/c mice. Methods: The presence of HCC was confirmed by histological and morphological alterations. In parallel to the incidence of hepatic cancer, we found lung, heart, and kidney cancer after treatment with DEN. Results: Our results show that the expression of mRNA of IQGAP1, TRAIL decoy receptors, NF- $\mathrm{KB}$, and IL-8 genes was elevated in hepatocellular carcinoma, as compared to normal liver tissue, while their expression was further up-regulated by increasing the dose of diethylnitrosamine. The expression of IQGAP2, TRAIL death receptors, p53, and PDCD5 was significantly down-regulated in HCC ( $\mathrm{p}<0.05)$. For confirmation of gene expression, protein levels of both IQGAP1 and P53 were measured by western blot analysis, which showed that diethylnitrosamine enhanced protein expression of IQGAP1 and diminished that of p53. Conclusion: IQGAPs have a direct signaling relationship with p53, IL-8, and TRAIL family. This interaction is recognized as a key signalling pathway for hepatocellular carcinogenesis.
\end{abstract}

Keywords: IQGAPs- IL-8- TRAIL- DEN- hepatocellular carcinoma

Asian Pac J Cancer Prev, 23 (1), 271-279

\section{Introduction}

$\mathrm{N}$-nitrosodiethylamine (DEN) is generally utilized as a carcinogenic agent and the main organ to be targeted is species-specific. Generally, following DEN administration, mice develop not only liver cancer but also gastrointestinal, skin, respiratory and haematopoietic cancers (Saber et al., 2018; Santos et al., 2017; Zhang et al., 2019). The carcinogenicity of "DEN" is due to its ability to alkylate DNA components. It elicits its effects in a dose-dependent manner (Liu et al., 2020). A single dose of DEN with low initiation does not result in the production of carcinoma, whereas administration of a higher dose can induce Hepatocellular carcinoma (HCC) after a latency period (Zoheir et al., 2015). HCC is known to be the fifth most common solid tumour in the world, and the third most common reason of cancer-related mortality (Sayiner et al., 2019). Due to the fact that most HCC patients have acquired cirrhosis, chemotherapy regimen or major resections are not efficiently tolerated (Wang et al., 2019).

Apoptosis is a unique mode of programmed cell death that is essential for cellular homeostasis in the normal tissues and is regularly suppressed in tumorigenic processes (Jan, 2019). Deregulations of apoptosis and accelerated proliferation of hepatocytes have been declared as crucial factors, with respect to carcinogenesis of the liver or tumour progression in HCC (Czauderna et al., 2019). Basically, apoptosis occurs in cells in response to binding of ligands to their specific death receptors (DRs) through the extrinsic pathway, or to a variety of intracellular, mitochondria-initiated, stress signals which act directly on targets inside the cells in what is known as the mitochondrial or intrinsic pathway. The binding of TNF related apoptosis-inducing ligand (TRAIL) to its death receptors (DR4 and DR5) or decoy receptors (DcR1 and DcR2) is a vital example of the process regulation (Micheau, 2018). Contrary to TRAIL, $\mathrm{NF}-\mathrm{kB}$ is an anti-apoptotic transcription factor that is often active in a constitutive manner in a number of cancers (Medeiros et al., 2021; Puar et al., 2018; Rasmi et al., 2020). NF-kB is actually known to induce the expression of the inflammatory CXC chemokine interleukin-8 (IL-8) in numerous cancers including HCC (Arvanitakis et al., 
2021).

Programmed cell death 5 (PDCD5), known also as TF-1 cell apoptosis-related gene-19 (TFAR19), was originally recognized in human leukemia TF-1 cells having serum withdrawal-induced apoptosis (Ha et al., 2021; Li et al., 2018). PDCD5 was declared downregulated in a lot of the human malignancies, including breast cancer (Hedenfalk et al., 2001) lung adenocarcinoma and hepatocellular carcinoma (Ma et al., 2019), as well as ovarian cancer (Gao et al., 2015). Accumulating evidence demonstrates that PDCD5 stimulates apoptosis of numerous human cancer cells, including TF-1, gastric cancer MGC-803, breast cancer, cervical cancer (Li et al., 2018).

We have recently shown that isoleucine-glutaminemotif containing GTPase-activating proteins (IQGAPs) have a direct and obvious signaling relation with RAS genes in production of cancer, and they are recognized as important target genes for regulation of the liver carcinogenesis (Zoheir et al., 2015). IQGAPs constitute a tiny subgroup of evolutionally preserved superfamily of GTPase-activating proteins which exist in eukaryotes ranging from yeast to the mammals. These proteins are generally abbreviated as IQGAPS due to their isoleucineglutamine (IQ) and GTP-activating protein (GAP) domains (Xiao et al., 2018).

In this current study, we sought to investigate the signaling crosstalk of TRAIL receptors, PDCD5, p53 with IQGAPs, NF-kB, and IL-8 and its receptors in an induced model of HCC.

\section{Materials and Methods}

\section{Ethics approval}

Animals were kept in compliance with the National Institutes of Health (NIH) Guide for the Care and Use of Laboratory Animals. Medical Research Ethics Committee approved this study "Project number"12060168".

\section{Animals}

A total of eighty 21-day-old male Balb/c mice were purchased from the Animal Care Center, and housed in polycarbonate cages in an animal facility accredited by the Association for Laboratory Animal Care Evaluation and Accreditation. All mice were fed NIH-07 diet (rodent chow) and received water ad libitum. Before the tests, the animals were allowed to acclimatize for a week. There were special training in animal care or handling provided for research staff. Every day frequently animal health and behavior were monitored to determine when animals display early markers associated with death or poor prognosis of quality of life, or specific signs of severe suffering or distress, and should be euthanized before allowing such conditions to continue or progress to death. Every day we frequently monitored the health of the animals, whether there were any unexpected deaths, and we determined cause of death in these cases.

\section{Chemicals}

DEN was bought from Sigma Chemicals (St. Louis, MO, USA). TRIzol reagent was brought from Invitrogen (Carlsbad, CA, USA). High-Capacity cDNA reverse transcription kit, primers for real time PCR, and SYBR Green Universal Master mix were all bought from Applied Biosystems $^{\circledR}$ (Foster City, CA, USA). Antibodies specific for b-actin, IQGAP1 and P53 were purchased from (Santa Cruz Biotechnology).

\section{Experimental Design}

After acclimation, the 4-week-old mice were randomly divided into six experimental groups, further divided into 4-week and 8-week groups. The 4-week experimental group of mice were injected intraperitoneally (i.p.) with DEN that was dissolved in saline at the exact following dosages of 0,50 and $75 \mathrm{mg} / \mathrm{kg}$, groups 1-3, respectively, during the experimental days number $0,7,14$ and 21 . The i.p. injections were given in the 8-week treatment group as described above on experimental days $0,7,14,21,28$, 35, 42 and 49 (groups 4-6). Mice were euthanized by $\mathrm{CO}_{2}$ asphyxiation and exsanguination, weighed and necropsied at experimental day 288 (after injection of DEN). The mice livers were removed in toto, weighed and examined to find if there was any grossly visible lesion. In addition, these livers were then divided by lobe and cut into $1-$ to $2-\mathrm{mm}$ slices. One piece from each lobe was fixed in formalin for a period of 48 to 72 hours and then was embedded in paraffin to finally give a total of three paraffin blocks per animal. Serial sections from each block were stained with hematoxylin and eosin (H\&E), and hepatic preneoplastic and neoplastic lesions were classified basically on the pathology of the mouse (16).

\section{Quantitative Analysis of Altered Hepatocellular Foci (AHF)}

Quantitative analysis of AHF was achieved by operating a two-dimensional evaluation method. Whole H\&E staining sections were scanned by ScanScope CS (Aperio Technologies, Vista, CA, USA). Images were then viewed with Aperio's ImageScope viewer software (Aperio Technologies), on which the quantitative analyses were performed. The number and volume of each foci (eosinophilic, clear and basophilic) and total volume of liver sections were quantified. Because large tumors of the liver were recognized in one animal (one animal in the $75 \mathrm{mg} / \mathrm{kg}$ for 8 weeks group), these areas were not included in the total volume of liver sections of this animal. The multiplicity of AHF was reported as numbers per centimeter squared, and the volume of foci was represented as the percentage of total liver volume (\%).

\section{RNA extraction and cDNA synthesis}

Under the manufacturer's instructions, the total RNA from the liver tissue homogenate was isolated using TRIzol reagent (Gibco, Invitrogen; Eugene, OR, USA) and RNA absorbance was measured at $260 \mathrm{~nm}$. The RNA quality was determined by measuring the $260 / 280$ ratio. According to the manufacturer's instructions, the first strand cDNA synthesis was operated by utilizing the High-Capacity cDNA reverse transcription kit (Applied Biosystems $^{\circledR}$; Foster City, CA, USA). 
Quantification of $m R N A$ expression by real-time polymerase chain reaction (RT-PCR)

To help identify the markers of apoptosis and inflammation, the Quantitative analyses of target genes mRNA expression were undertaken by RT-PCR. Briefly, the resultant cDNA from the above preparation was subjected to PCR amplification using 96-well optical reaction plates in the ABI Prism 7500 System (Applied Biosystems). The $25 \mu \mathrm{l}$ reaction mixture contained $0.1 \mu \mathrm{l}$ of $10 \mu \mathrm{M}$ forward primer and $0.1 \mu \mathrm{l}$ of $10 \mu \mathrm{M}$ reverse primer (40 $\mathrm{nM}$ final concentration of each primer), 12.5 $\mu 1$ of SYBR Green Universal Master mix, $11.05 \mu 1$ of nuclease-free water and $1.25 \mu 1$ of cDNA sample. The primers that have been used in the study were chosen from the website: pubmed.com (http://www.ncbi.nlm.nih. gov/tools/primer-blast) as listed in Table 1. To test for the contamination of any assay reagents, controls of the assay, namely, no-template controls, were incorporated onto the same plate,. The real-time PCR data were analyzed utilizing the relative gene expression (i.e., $\Delta \Delta \mathrm{CT}$ ) method, as described in Applied Biosystems User Bulletin No. 2. The data are presented as the fold change in gene expression normalized to the endogenous reference gene glyceraldehyde-3- phosphate-dehydrogenase (GAPDH) and relative to a calibrator.

\section{Western blot analysis}

The hepatocytes were homogenized and lysed in RIPA buffer (50 mM Tris-HCI, pH 8.0, $150 \mathrm{mM}$ sodium chloride, $1.0 \%$ NP-40, $0.5 \%$ sodium deoxycholate, $0.1 \%$ sodium dodecyl sulfate (SDS) and $0.1 \%$ of protease inhibitor cocktail) for $20 \mathrm{~min}$ at $40 \mathrm{C}$. The lysates were centrifuged at $14,000 \mathrm{rpm}$ at $40 \mathrm{C}$ for $15 \mathrm{~min}$ to remove debris. Protein concentrations of whole cell lysates were determined using the Protein Assay Kit (Sigma Aldrich, Catalog Numbers BCA1 and B9643). To prepare $50 \mathrm{mg}$ of each sample taken, the supernatant protein has been isolated by SDS-10 percent polyacrylamide gel electrophoresis (SDS-PAGE) and transferred, by standard procedures, to polyvinylidene difluoride (PVDF) membrane (Bio-rad, Hercules, CA, USA). The membranes were hybridized with primary antibodies followed by incubation with appropriate secondary antibodies. By treatment with the chemi-luminescence detection reagent (Amersham Biosciences), according to manufacturer's instructions, the antibody-bound proteins were observed after exposure to X-ray films (Kodak X-Omat). The same membranes were re-probed with anti- $\beta$ actin antibody, which was used as an internal control for protein loading.

IQGAP1 and P53 antibodies as well as those rose against $\beta$-actin were diluted in PBST containing 5\% non-fat milk and incubated overnight with membranes at $4^{\circ} \mathrm{C}$. The blot was incubated for one hour at room temperature, after already being washed with an anti-mouse or anti-rabbit IgG accompanied with horseradish peroxidase (Millipore, 1:5000 dilutions in PBST containing 5\% non-fat milk). Finally, the blots were visualized using a chemiluminescence detection kit (ECL; Millipore), and the densitometric scanning has quantified the optical density of each band.

\section{Histopathological Examination}

Liver morphology of Balb/c mice either normal or DEN-treated ones was examined by histopathology. Liver sections (5 micron-thickness) were fixed and stained with regular Hematoxylin-eosin stain (H\&E), and then examined under light microscope and lesions were photographed with Olympus digital camera installed on the Olympus microscope with $1 / 2 \mathrm{X}$ photo-adaptor, using 100X objective lens.

\section{Enzyme-linked immunosorbent assay (ELISA)}

Tumor necrosis factor (TNF)- $\alpha$ and interleukin (IL) $-1 \beta$ in the mouse serum were measured according to the manufacturer's specifications (R\&D Systems, Minneapolis,

$\mathrm{MN}, \mathrm{USA})$. The OD value at $450 \mathrm{~nm}$ was determined by a Power Wave microplate reader Bio-Tek, USA.

\section{Flow Cytometer Analysis}

This analysis was performed using Annexin V FITC and Propidium Iodide (PI), in order to measure the rates of apoptosis in blood cells. Annexin V-stained flipped phospholipids in lymphocytes cell membranes and Propidium Iodide (PI)-stained that damaged DNA were used in determination of early and late apoptosis, respectively, after treatment compared to control. Annexin $\mathrm{V}$ and PI were purchased from (Thermo Fisher Scientific). The apoptotic analysis was dedicated to differentiate between early and late apoptotic cells, as well as necrotic cells. The Apoptosis of the treated and untreated cells was analyzed by flow cytometer instrument (Attune ${ }^{\circledR}$ Acoustic Focusing Cytometer, Life Technologies) and analyzed by its software.

\section{Results}

Mortality and occurrence of liver, lung and heart cancer or injury liver

We found high percentage of mortality (deal animals) in treated animals with DEN in either 4 or 8 - weeks' treatment. In addition we found occurrence of lung and heart cancer in treated animals with DEN parallel to liver cancer (Table 2).

\section{Histopathological Examination}

Histopathology (Figures 1-2) revealed dose-dependent degenerative progression in hepatocyte structure, since administration of DEN in a dose of $50 \mathrm{mg} / \mathrm{Kg}$ once weekly i.p for 4 weeks showed fatty degeneration and scattered inflammatory cell infiltrate. While, the higher dose 75 $\mathrm{mg} / \mathrm{Kg}$ for the same period of time showed steatohepatitis with fatty degeneration and scattered inflammatory cell infiltrate. Higher dose of DEN (75 mg/Kg) i.p once weekly for 8 weeks showed HCC that comprises sheets and nests of large tumor cells with pleomorphic nuclei, prominent nucleoli, abundant granular eosinophilic cytoplasm, scatter Mallory bodies (dense eosinophilic cytoplasmic structure) and distinct cell borders. 
Table 1. Primer Sequences of Used Mouse Genes

\begin{tabular}{|c|c|}
\hline \multirow[t]{2}{*}{ NM_011399. 2 Mus muscullus:1L-8 } & F: CTAGGCATCTTCGTCCGTCC \\
\hline & R: AGCCCATAGTGGAGTGGGAT \\
\hline \multirow[t]{2}{*}{ NM_009910.3 Mu muscullus: Cxcr3B } & F: TCAGCCAACTACGATCAGCG \\
\hline & R: CCTCTGGAGACCAGCAGAAC \\
\hline \multirow[t]{2}{*}{ Mus muscullus:DCR1 } & F: GCTGAAGAGACAATGAAC \\
\hline & R: ACGATCACAAGGAGGAAG \\
\hline \multirow[t]{2}{*}{ AY521463.1 Mus muscullus:Nfkb1 } & F: ACTGTCTGCCTCTCTCGTCT \\
\hline & R: AAATGTAAAATGCATAAAACGGGGA \\
\hline \multirow[t]{2}{*}{ AB020317.1 Mus muscullus:P53 } & F: GCCCATGCTACAGAGGAGTC \\
\hline & R: TGAGTGGAATCTGGGATTGTG \\
\hline \multirow[t]{2}{*}{ NM_019746.4 Mus muscullus:Pdcd5 } & F: GGACTCCGATGAAGACGACG \\
\hline & R: GACTGTCCTAGACACTGCTCC \\
\hline \multirow[t]{2}{*}{ NM_020275.4 Mus muscullus: Tnfrf10b (DR5) } & F: CACTGACGGGGAAGAGGAAC \\
\hline & R: TCGTCAGCTGAGTCGTTTCC \\
\hline \multirow[t]{2}{*}{ NM_178241.4 Mus musculus: Cxcr1 } & F: CAGCTGGTGCCTCAGATCAAA \\
\hline & R: CCAAGAAGGGCGGTCAAT \\
\hline \multirow[t]{2}{*}{ NM_009909.3Mus musculus: Cxcr2 } & F: ATGGCCTTGTCAGCAAGGAG \\
\hline & R: CTTCTACACGAACTCCCCACC \\
\hline \multirow[t]{2}{*}{ NM_003844.3 Mus musculus: DR4 } & F: ATGAACTCACTGGTTTCTTGGC \\
\hline & R: TTCGCGTCCGGCTTCCTCAAG \\
\hline \multirow[t]{2}{*}{ Mus muscullus:DCR2 } & F: AGCTGTGGTTGTGGTTGG \\
\hline & R: GGGTCAAGTACTGGACTG \\
\hline NM_016721.2 Mus musculus Iqgap1 & F: TTCAAGGTCTCTGCGCTTCC \\
\hline NM 027711.1 Mus musculus Iqgap2 & R: GAGGCCGGTAGCCTTGTATC \\
\hline
\end{tabular}

DEN stimulates expression of IQGAP1, TRAIL decoy receptors, NFKB and $I L-8$

The genetic expression of IQGAP1, as well as the antiapoptotic genes TRAIL decoy receptors DcR1 and DcR2, NF-kB and IL- 8 and its receptors CXCR1 and CXCR2 was significantly higher $(\mathrm{P}<0.001)$ in $\mathrm{DEN}$-induced cancer groups, as compared to their corresponding normal control ones (Figures 3a- d and 4a). Furthermore, the expression of these genes was increased by increasing the DEN dose.

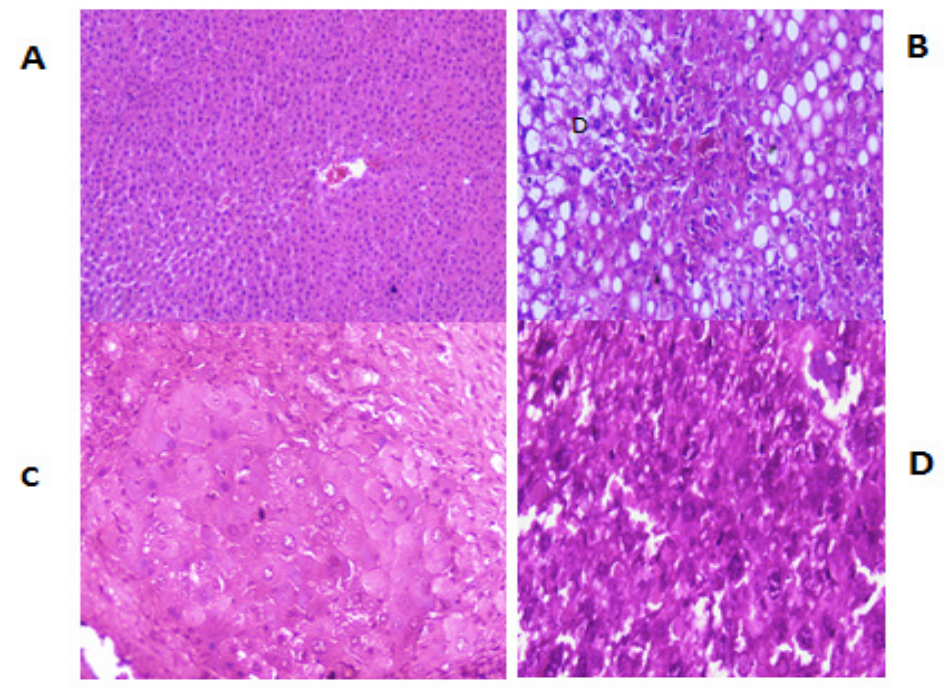

Figure 1. Histopathological Examination. A, Normal looking of controlled mouse hepatocytes (H\&E stain,100x); $\mathrm{B}$, Steatohepatitis of mouse hepatocytes treated with DEN $50 \mathrm{mg} / \mathrm{kg}$ once weekly i.p for 4 weeks showing fatty degeneration and scattered inflammatory cell infiltrate (H\&E stain, 200x); C, Hepatocytes from another rat treated with DEN $75 \mathrm{mg} / \mathrm{kg}$ once weekly i.p for 4 weeks show steatohepatitis, fatty degeneration and scattered inflammatory cell infiltrate (H\&E stain, 200x); D, Liver of mice treated with DEN $75 \mathrm{mg} / \mathrm{kg}$ i.p once weekly for 8 weeks shows HCC that comprises sheets and nests of large tumor cells with pleomorphic nuclei, prominent nucleoli, abundant granular eosinophilic cytoplasm, scattered Mallory bodies (dense eosinophilic cytoplasmic structures) and distinct cell borders (H\&E stain, 200x). 


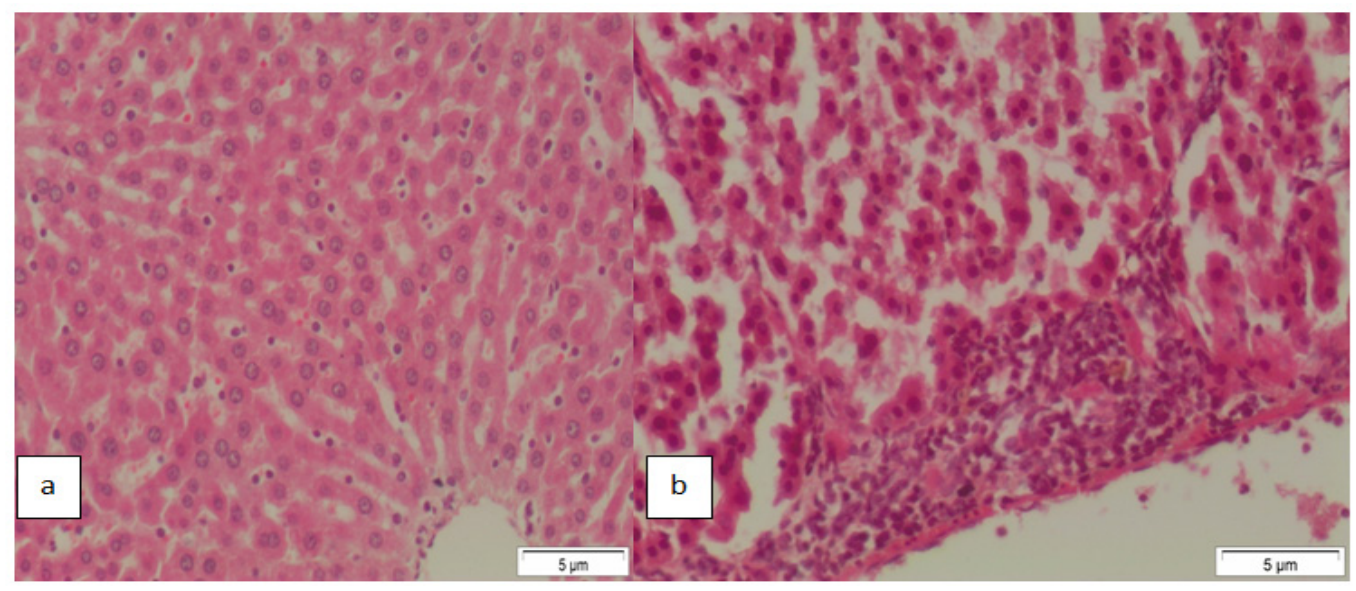

Figure 2. a, A micrograph of section of liver of control mouse shows the architecture of the hepatic lobule. The hepatocytes showed strongly eosinophilic granulated cytoplasm, and round nuclei with peripherally dispersed chromatin and prominent nucleoli. Between the strands of hepatocytes the hepatic sinusoids are often seen. The Kupffer cells are satellite and site on the sinusoidal surface of the endothelial cell, projecting in the vascular space (H \& E. stain, Scale bar: $5 \mu \mathrm{m})$; b, A micrograph of section of liver of DEN mouse liver showing hepatocellular carcinoma. It shows trabeculae of malignant hepatocytes separated by sinusoids (H \& E. stain, Scale bar: $5 \mu \mathrm{m}$ ).

DEN inhibits expression of IQGAP2, TRAIL death receptors, $p 53$ and PDCD5

The genetic expression of IQGAP2, as well as the pro-apoptotic genes TRAIL death receptors DR4 and DR5, p53 and PDCD5 was significantly lower $(\mathrm{P}<0.001)$ in all animal treated with DEN as compared to their corresponding normal control groups and their expressions were further decreased by increasing the DEN dose as shown in Figures 3a- d and 4a).

\section{Protein analysis}

Protein level of IQGAP1 showed a highly significant increase in DEN-treated group (4 weeks) as compared to normal controls while $\mathrm{p} 53$ protein level was significantly decreased in DEN-treated group as compared to the control (Figure 4b-c).

\section{Enzyme-linked immunosorbent results}

Both of tumor necrosis factor (TNF)- $\alpha$ and interleukin (IL)-1 $\beta$ were highly significant increase in both treated animals with DEN if compared with negative control.

Detection of early and late apoptosis by Flow Cytometer

All treated animals with DEN either 4 or 8 -weeks showed high incidence of early and late apoptosis if compared with negative control. In 8- weeks treated group, there were significant increases in necrotic cells if compared to 4- weeks treated group (Figure 5).

\section{Discussion}

IQGAP family of proteins is known to possess various protein-protein interaction domains. These members control certain cellular processes, including cell adhesion and migration, cytokinesis and many extracellular signals. Three IQGAPs occur in human beings: IQGAP1 and IQGAP2. IQGAP1 is this family's best characterized protein and is known to be over-expressed in HCC (Zoheir et al., 2015). This IQGAP1 has a domain of calponin homology, a domain of polyproline, and a RasGAP-related domain (GRD), as well as a sequence of RasGAP carboxyterminal that is common to members of the IQGAP family (Brown and Sacks, 2006). IQGAP1 assists in both ERKand $\beta$-catenin-dependent signaling. IQGAP1 binds B-Raf, MEK and ERK to facilitate their sequential activation and propagation of the MAPK cascade. Constitutive activation of the MAPK pathway is a common oncogenic trigger in several cancers (White et al., 2009).

The process of programmed cell death is initiated in cells in response to either extracellular cues via stimulation of the death receptors (DRs), often referred to as the extrinsic pathway, or to a variety of intracellular stress conditions that involve mitochondria and are known as the intrinsic pathway. A distinct mode of programmed apoptosis is critical for tissue homeostasis and its deregulation is frequent in malignancy. The extrinsic pathway starts with binding of death ligands to

Table 2. Showing Percentages (\%) of Mortality and Occurrence of Liver, Lung and Heart Cancer or Injury Liver

\begin{tabular}{lccc}
\hline Trial & $\begin{array}{c}\text { Normal Control } \\
(20 \text { male rats })\end{array}$ & $\begin{array}{c}\text { DEN positive control } \\
(40 \text { male rats) (4 weeks) }\end{array}$ & $\begin{array}{c}\text { DEN positive control } \\
(40 \text { male rats })(8 \text { weeks })\end{array}$ \\
\hline Number of dead animals (Mortality) & $1 / 20(2.5)$ & $10 / 40(25)$ & $12 / 40(50)$ \\
Presence of cancer by Alpha-fetoprotein (AFP) analysis & $0 / 20(0)$ & $25 / 40(62.5)$ & $27 / 40(67.5)$ \\
Liver cancer by histopathology observation & $0 / 20(0)$ & $25 / 40(62.5)$ & $27 / 40(67.5)$ \\
Presence of liver injury & $0 / 20(0)$ & $5 / 40(12.5)$ & $1 / 40(2.5)$ \\
Presence of lung cancer & $0 / 20(0)$ & $25 / 40(62.5)$ & $27 / 40(67.5)$ \\
Presence of heart cancer & $0 / 20(0)$ & $15 / 40(37.5)$ & $16 / 40(40)$ \\
\hline
\end{tabular}



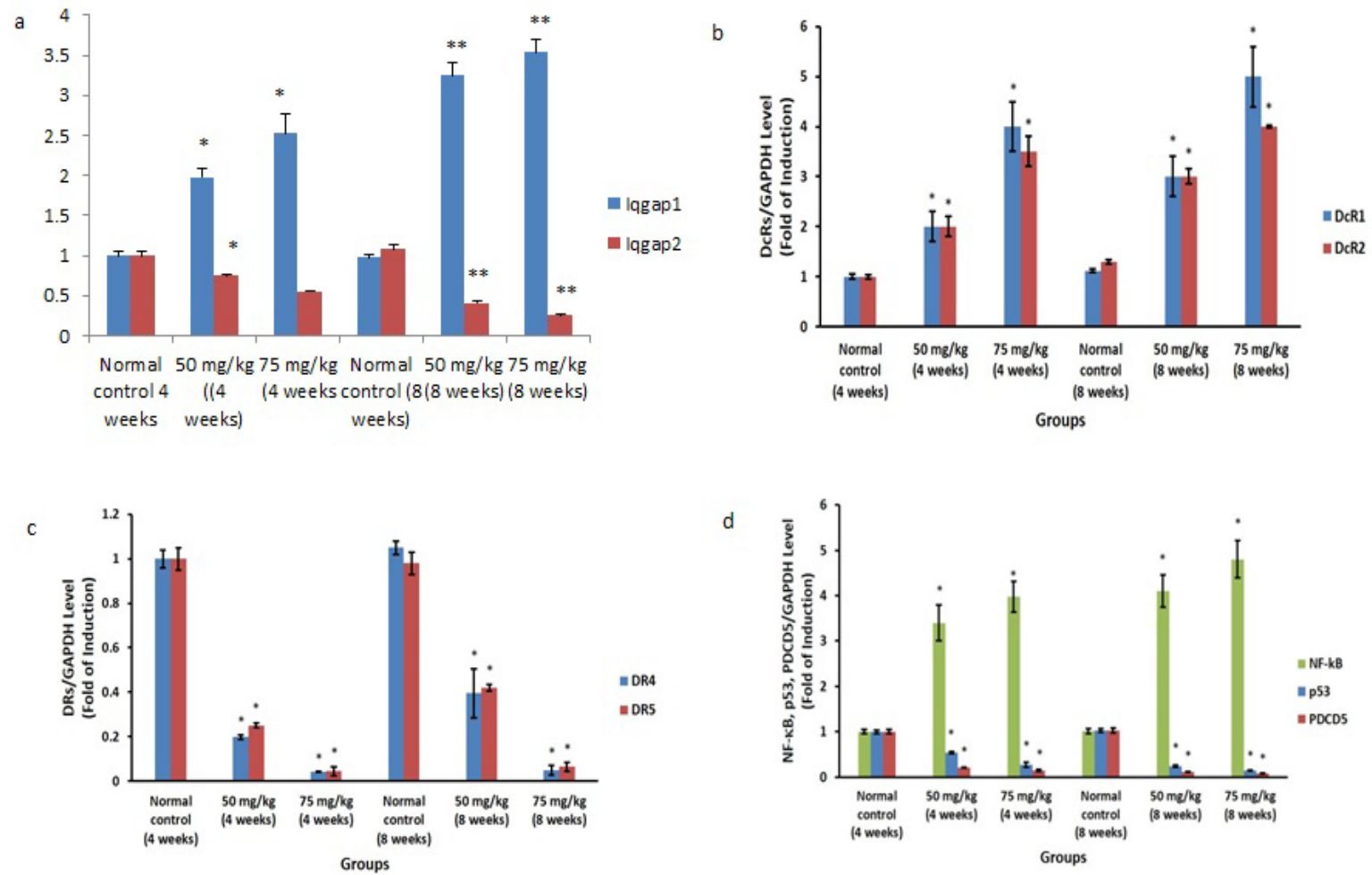

Figure 3. DEN Regulates Expression of IQGAPs, DcR1 and DcR2, DR4, DR5 and NF-ng Genes in Liver Tissues. a, mRNA amounts of IQGAP1 and 2 were quantified using RT-PCR and normalized to GAPDH housekeeping gene as in Methods; $b$,) mRNA amounts of DcR1 and DcR2 were quantified using RT-PCR and normalized to GAPDH housekeeping gene as in Methods. Statistical analysis was performed using ANOVA followed by t-test. Each value indicates the mean \pm S.E.M of ten animals. $p<0.05$ was considered significant $(*)$ compared to the corresponding normal control $(0 \mathrm{mg} / \mathrm{kg})$ group.
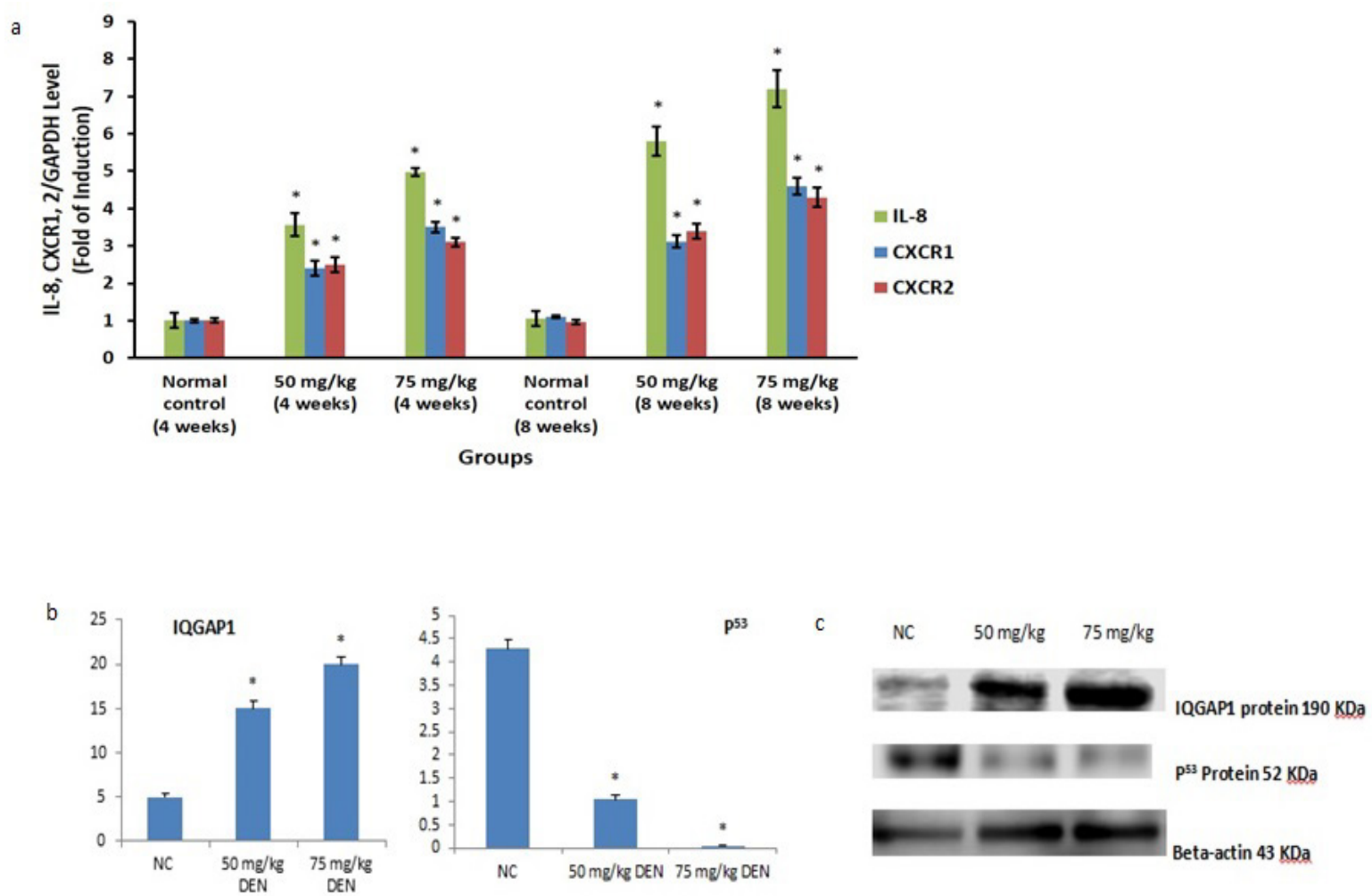

Figur 4. a, DEN suppresses expression of IL-8, CXCR1 and CXCR2 genes in liver tissues; b and c, DEN upregulates IQGAP1 and downregulates $\mathrm{p}^{53}$ expression in DEN-treated liver cells. Balb/c mice were treated with noted concentrations of DEN 4 weeks. Liver tissues were homogenized and total protein was prepared as described in Methods. IQGAP1 and $\mathrm{p}^{53}$ protein levels were determined by Western blot analysis (upper panel). Representative densitometric quantifications of IQGAP1 and $\mathrm{p}^{53}$ protein levels are shown in the lower two panels. Values are presented as means \pm standard error of the mean. ${ }^{*} \mathrm{p}<0.05$, compared with control (NC). 


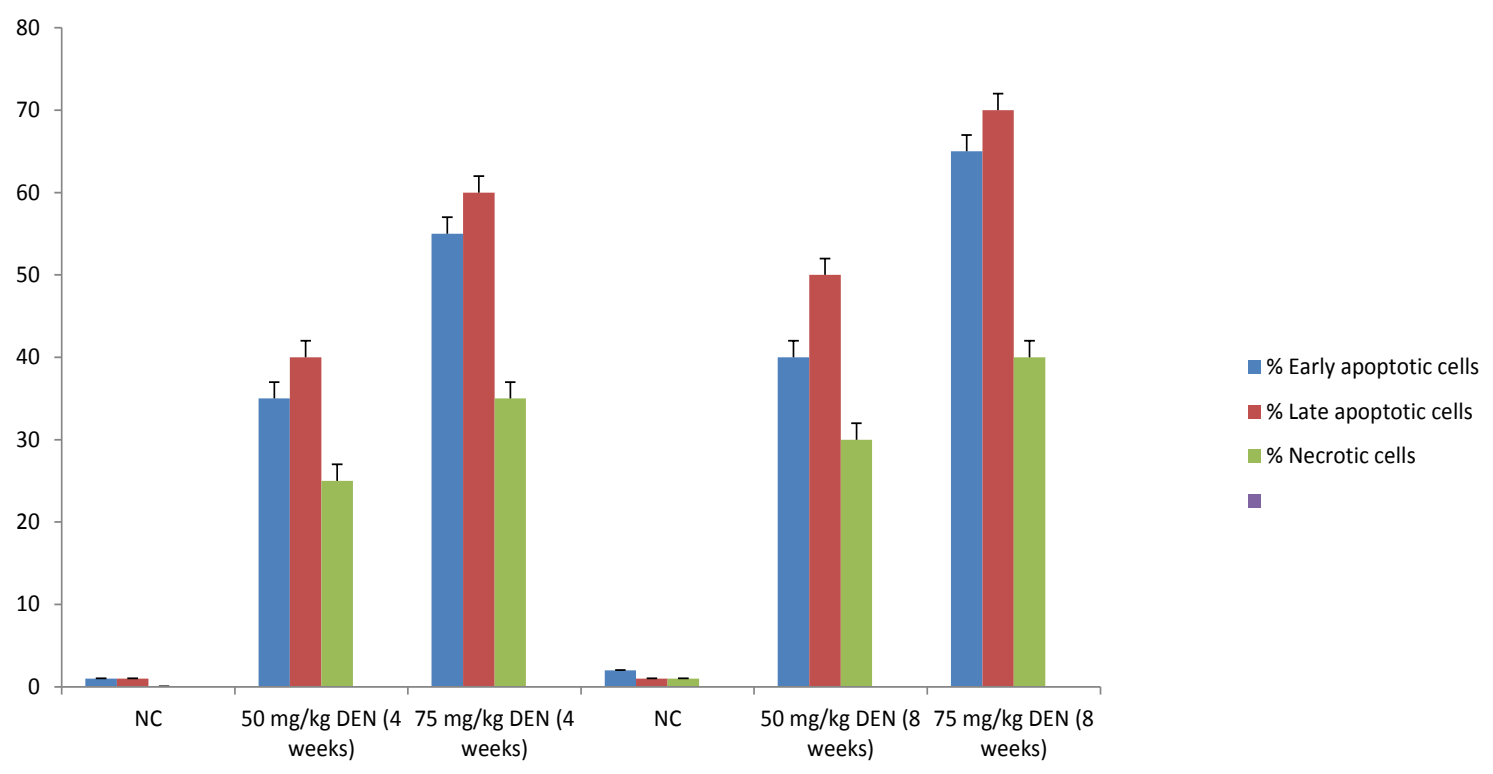

Figure 5. Flow Cytometry Analysis Using Annexin V FITC and Propidium Iodide (PI) for Apoptosis Measurements. Blood cells were treated with DEN. Early, late apoptosis, and necrosis were detected in the stained cells with Annexin $\mathrm{V}$ and PI .

their death receptors such as the ligand tumor necrosis factor-related apoptosis-inducing ligand (TRAIL) to its membrane-bound death receptors [DR4 and DR5] (Mehdi et al., 2019). This induces the aggregation of receptors and the recruitment of the Fas-associated death domain (FADD) and caspase-8. The later then becomes activated and initiates apoptosis by activation of the downstream effector caspase-3, resulting in caspase-3-mediated poly (ADP-ribose) polymerase (PARP) cleavage and other morphological features linked to apoptosis (Fulda and Debatin 2006).

Interleukin-8 (IL-8), a member of the CXC chemokine family, is overexpressed in HCC compared with normal liver, and a high serum IL-8 level is significantly correlated with a more aggressive tumor phenotype (Ren et al., 2003). IL-8 has been shown to block TRAIL-induced cell death in ovarian cancer (Abdollahi et al., 2003). TRAIL is known to induce apoptosis in a wide variety of cancer cells, including HCC cells, but rarely in normal cells. Normal cells are thought to be resistant to TRAIL because their cell surface has the ability to express higher levels of TRAIL decoy receptors DcR1 and/or DcR2 (MacFarlane 2003 and Abdel Salam et al., 2021). Human HCC cells have been demonstrated to display high resistance to TRAILmediated cell death. However, TRAIL anti-tumor activity against HCC has not been thoroughly investigated and the mechanisms of such resistance to TRAIL remain unclear (Abd-Rabou et al., 2021).

The genes of IQGAPs play a crucial role in the induction or suppression of cancer and its progression; but their relationship with IL-8and TRAIL, and their receptors, is still uncertain. The goal of this study was therefore to evaluate the genetic expressions of the IQGAP family (IQGAP1, IQGAP2 and IQGAP3), IL-8 and TRAIL receptors, NF-kB, p53 and PDCD5 in mice with DEN-induced HCC and normal controls.
According to our results, the expression level of IQGAP1 was significantly upregulated in HCC tissues, compared to normal liver tissue. This corroborates previous findings by Chen et al., (2010), who reported that IQGAP1 is overexpressed in HCC. Our data also show that NF-kB and IL-8 and its receptors were upregulated in HCC tissues, as compared to normal ones. These data confirm previous findings showing that NF-kB (He and Karin, 2011) and IL-8 (Ren et al., 2003) are upregulated in HCC. Knowing that IQGAP1 triggers activation of NF-kB (Tseng et al., 2014), our data reveals that IQGAP1 stimulates NF-kB signaling, which in turn, augments expression of its downstream effectors IL-8 and its receptors CXCR1 and CXCR2. Notably, to the best of our knowledge, we are the first group to report overexpression of IL-8 receptors in DEN-induced HCC and the first group to report overexpression of CXCR2 in HCC, in general. Moreover, we report, for the first time, that DEN activates such an IQGAP1/ NF-kB/IL-8 pathway in HCC in vivo.

The pro-apoptotic TRAIL death receptors were markedly downregulated in HCC cells, as compared to normal liver cells. In addition, DEN induced upregulation of TRAIL decoy receptors. This suggests that this regulation of TRAIL receptors facilitated DEN-induced mutagenicity and carcinogenicity. Since our data showed that DEN stimulated NF-kB and IL-8 expression, and NF-kB and IL-8 are known to suppress TRAIL-induced apoptosis, we may conclude that DEN fostered NF-kB expression, which in turn upregulated IL-8 expression and then both NF-kB and IL-8 suppressed TRAIL death receptors expression. In addition, TRAIL death receptors are known to be transcriptionally upregulated by p53 (Willms et al., 2019). Since p53 has been shown to inhibit NF-kB activity (Shao et al., 2000) and our data reveal that DEN significantly decreases p53 mRNA and protein levels, this could allow for upregulation NF-kB, 
which in turn could stimulate IL- 8 signalling and inhibit TRAIL death receptors expression. Furthermore, since $\mathrm{NF}-\mathrm{kB}$ is known to upregulates TRAIL decoy receptors (Bernard et al., 2001), we may conclude that DEN-induced upregulation of NF-kB may have augmented expression of TRAIL decoy receptors.

Our data showed that PDCD5 was downregulated in HCC tissues, relative to normal liver tissue. This agrees with findings by $\mathrm{Fu}$ et al., (2013) who reported that expression of PDCD5 was lower in HCC tissue compared to normal tissue. Knowing that PDCD5 is an important upstream regulator of P53 which upregulates p53 (Fu et al. 2013), we may conclude that DEN-induced PDCD5 downregulation in $\mathrm{HCC}$ resulted in diminished expression of p53, which then allowed for stimulation of the IQGAP1/ NF-kB /IL-8 pathway.

In conclusion, our DEN mouse model for liver cancer clearly showed that that there is a crosstalk between these two pathways, namely IQGAP1/NF-kB/IL-8 and PDCD5/ p53/TRAIL/caspase-8/caspase-3 pathways. Occurrence of apoptosis and cell death and necrosis were confirmed via other parameters such as flow cytometer and tumor necrosis factor (TNF)- $\alpha$ and interleukin (IL)-1 $\beta$. In addition, DEN made lung and heart cancer parallel to liver cancer which will be the new topic for further manuscript.

\section{Author Contribution Statement}

All authors participated in the design, interpretation of the studies and analysis of the data and review of the manuscript; $\mathrm{KZ}$ designed this study. KM, MA, AD, and $\mathrm{KZ}$ participated in the conduct of the study. MA, AD, KM and $\mathrm{K} . \mathrm{Z}$ conducted the experiments, $\mathrm{KZ}$ supplied critical reagents, $\mathrm{KZ}$ wrote the manuscript.

\section{Acknowledgments}

All authors thank National Research Centre Supporting Project, National Research Centre, Dokki, Egypt.

\section{Ethics Approval and Consent to Participate}

The experimental protocol used in the study was approved (NRCE-CBD1032019) by the Animal Care and Use Committee of National Research Centre, Egypt.

Availability of data and material

Data is available for all.

\section{Competing interests}

There is no competing interest.

\section{Funding}

This study was funded by National Research Centre Supporting Project number (NRC-12060168), National Research Centre, Dokki, Egypt.

\section{References}

Abdel Salam N, Abd-Rabou A, Sharada H, EL Samea G, Abdalla M (2021). Combination therapy of TRAIL and thymoquinone induce breast cancer cell cytotoxicity- mediated apoptosis and cell cycle arrest. Asian Pac J Cancer Prev, 22, 1513-21.

Abdollahi T, Robertson NM, Abdollahi A, Litwack G (2003). Identification of interleukin 8 as an inhibitor of tumor necrosis factor-related apoptosis-inducing ligand-induced apoptosis in the ovarian carcinoma cell line OVCAR3. Cancer Res, 63, 4521-6.

Abd-Rabou A, Abd El-Salam N, Sharada H, Abd EL Samea G, Abdalla M (2021). Thymoquinone crosstalks with DR5 to sensitize TRAIL resistance and stimulate ROS-mediated cancer apoptosis. Asian Pac J Cancer Prev, 22, 2855-65.

Arvanitakis K, Mitroulis I, Germanidis G (2021). Tumorassociated neutrophils in hepatocellular carcinoma pathogenesis, prognosis, and therapy. Cancers, 13, 2899.

Bernard D, Quatannens B, Vandenbunder B, Abbadie C (2001). Rel/NF-kappaB transcription factors protect against tumor necrosis factor (TNF)-related apoptosis-inducing ligand (TRAIL)-induced apoptosis by up-regulating the TRAIL decoy receptor DcR1. J Biol Chem, 276, 27322-8.

Czauderna C, Castven D, Mahn FL, Marquardt JU (2019). Context-dependent role of NF- $\mathrm{kB}$ signaling in primary liver cancer-from tumor development to therapeutic implications. Cancers, 11, 1053.

Chen F, Zhu HH, Zhou LF, et al (2010). IQGAP1 is overexpressed in hepatocellular carcinoma and promotes cell proliferation by Akt activation. Exp Mol Med, 42, 477-83.

Fu DZ, Cheng Y, He H, Liu HY, Liu YF (2013). PDCD5 expression predicts a favorable outcome in patients with hepatocellular carcinoma. Int J Oncol, 2013, 821-30.

Fulda S, Debatin KM (2006). Extrinsic versus intrinsic apoptosis pathways in anticancer chemotherapy. Oncogene, 25, 4798-4811.

Gao L, Ye RQ, Ma HY, et al (2015). Chang. Low programmed cell death 5 expression is a prognostic factor in ovarian cancer. Chin Med J, 128, 1084-90.

Ha F, Li N, Long C, et al (2021). The effect of global DNA methylation on PDCD5 expression in the PBMC of occupational chromate exposed workers. J Occupational Environ Med, 63, 600-8.

He G, Karin M (2011). NF- $\mathrm{BB}$ and STAT3 - key players in liver inflammation and cancer. Cell Res, 21, 159-68.

Hedenfalk I, Duggan D, Chen Y, et al (2001). Gene-expression profiles in hereditary breast cancer. $N$ Engl J Med, 344, 539-48.

Jan R (2019). Understanding apoptosis and apoptotic pathways targeted cancer therapeutics. Adv Pharm Bull, 9, 205.

Li P, Fei H, Wang L, et al (2018). PDCD5 regulates cell proliferation, cell cycle progression and apoptosis. Oncol Lett, 15, 1177-83.

Liu K, Chen J, McCaughan GW (2020). Animal models for hepatocellular carcinoma arising from alcoholic and metabolic liver diseases. Hepatoma Res, 28, 6.

Ma Z, Guo D, Wang Q, et al (2019). Lgr5-mediated p53 Repression through PDCD5 leads to doxorubicin resistance in Hepatocellular Carcinoma. Theranostics, 9, 2967.

MacFarlane M (2003). TRAIL-induced signaling and apoptosis. Toxicol Lett, 139, 89-97.

Medeiros M, Candido MF, Valera ET, Brassesco MS (2021). The multifaceted NF-kB: are there still prospects of its inhibition for clinical intervention in pediatric central nervous system tumors?. Cell Mol Life Sci, 78, 6161-200.

Mehdi SH, Zafaryab M, Nafees S, et al (2019). Chrysin sensitizes human lung cancer cells to tumour necrosis factor related apoptosis-inducing ligand (TRAIL) mediated apoptosis. Asian Pac J Cancer Biol, 4, 27-33.

Micheau O(2018) Regulation of TNF-related apoptosis-inducing ligand signaling by glycosylation. Int J Mol Sci, 19, 715 . 
Rasmi RR, Sakthivel KM, Guruvayoorappan C (2020). NF-кB inhibitors in treatment and prevention of lung cancer. Biomed Pharm, 130, 110569.

Puar YR, Shanmugam MK, Fan L, et al (2018). Evidence for the involvement of the master transcription factor NF- $\kappa B$ in cancer initiation and progression. Biomedicines, $\mathbf{6}, 82$.

Ren Y, Poon RT, Tsui HT, et al (2003). Interleukin-8 serum levels in patients with hepatocellular carcinoma: correlations with clinicopathological features. Clin Cancer Res, 9, 5996-6001.

Saber S, Mahmoud A, Helal N, El-Ahwany E, Abdelghany $R$ (2018). Liver protective effects of renin-angiotensin system inhibition have no survival benefits in hepatocellular carcinoma induced by repetitive administration of diethylnitrosamine in mice. Open Access Macedonian $J$ Med Sci, 6, 955.

Santos NP, Colaco AA, Oliveira PA (2017). Animal models as a tool in hepatocellular carcinoma research: A Review. Tumor Biol, 39, 1010428317695923.

Sayiner M, Golabi P, Younossi ZM (2019) Disease burden of hepatocellular carcinoma: a global perspective. Dig Dis Sci, 64, 910-7.

Shao J, Fujiwara T, Kadowaki Y, et al (2000). Overexpression of the wild-type p53 gene inhibits NF-kappaB activity and synergizes with aspirin to induce apoptosis in human colon cancer cells. Oncogene, 19, 726-36.

Tseng PC, Chen CL, Shan YS, et al (2014). An increase in integrin-linked kinase non-canonically confers NF- $\kappa \mathrm{B}$ mediated growth advantages to gastric cancer cells by activating ERK1/2. Cell Commun Signal, 12, 69.

Wang H, Lu Z, Zhao X (2019). Tumorigenesis, diagnosis, and therapeutic potential of exosomes in liver cancer. $J$ Hematol Oncol, 12, 1-21.

Wang L, Wang C, Su B, et al (2013). Recombinant human PDCD5 protein enhances chemosensitivity of breast cancer in vitro and in vivo. Biochem Cell Biol, 91, 526-31.

White CD, Brown MD, Sacks DB (2009). IQGAPs in cancer: a family of scaffold proteins underlying tumorigenesis. FEBS Lett, 583, 1817-24.

Willms A, Schittek H, Rahn S, et al (2019). Impact of p53 status on TRAIL-mediated apoptotic and non-apoptotic signaling in cancer cells. PLoS One, 14, e0214847.

Xiao XH, Lv LC, Duan J, et al (2018). Regulating Cdc42 and its signaling pathways in cancer: small molecules and MicroRNA as new treatment candidates. Molecules, 23, 787.

Zhang HE, Henderson JM, Gorrell MD (2019). Animal models for hepatocellular carcinoma. Biochim Biophy Acta (BBA)Mol Basis Dis, 1865, 993-1002.

Zoheir KM, Abd-Rabou AA, Harisa GI, et al (2015). Gene expression of IQGAPs and Ras families in an experimental mouse model for hepatocellular carcinoma: mechanistic study of cancer progression. Int J Clin Exp Pathol, 8, 8821-31.

\section{c) (7) (8)}

This work is licensed under a Creative Commons AttributionNon Commercial 4.0 International License. 